\title{
Physical Activity Patterns Using Accelerometry in the National Weight Control Registry
}

\author{
Victoria A. Catenacci ${ }^{1}$, Gary K. Grunwald ${ }^{1}$, Jan P. Ingebrigtsen ${ }^{1}$, John M. Jakicic ${ }^{2}$, Michael \\ D. McDermott ${ }^{2}$, Suzanne Phelan ${ }^{3}$, Rena R. Wing ${ }^{4}$, James O. Hill ${ }^{1}$, and Holly R. Wyatt ${ }^{1}$ \\ ${ }^{1}$ Center for Human Nutrition, University of Colorado Denver, Denver, Colorado, USA \\ ${ }^{2}$ Department of Health and Physical Activity, Physical Activity and Weight Management Research \\ Center, University of Pittsburgh, Pittsburgh, Pennsylvania, USA \\ ${ }^{3}$ Department of Kinesiology, California Polytechnic State University, San Luis Obispo, California, \\ USA \\ ${ }^{4}$ Department of Psychiatry and Human Behavior, Warren Alpert Medical School of Brown \\ University, Brown University, Providence, Rhode Island, USA
}

\begin{abstract}
The National Weight Control Registry (NWCR) was established in 1993 to examine characteristics of successful weight-loss maintainers. This group consistently self-reports high levels of physical activity. The aims of this study were to obtain objective assessments of physical activity in NWCR subjects and compare this to physical activity in both normal-weight and overweight controls. Individuals from the NWCR $(n=26)$ were compared to a never-obese normal-weight control group matched to the NWCR group's current BMI $(n=30)$, and an overweight control group matched to the NWCR group's self-reported pre-weight-loss BMI ( $n=$ 34). Objective assessment of physical activity was obtained for a 1-week period using a triaxial accelerometer. Bouts of moderate-to-vigorous physical activity (MVPA) $\geq 10 \mathrm{~min}$ in duration, as well as nonbout MVPA (bouts of MVPA 1-9 min in duration) were summed and characterized. NWCR subjects spent significantly $(P=0.004)$ more time per day in sustained bouts of MVPA than overweight controls $(41.5 \pm 35.1 \mathrm{~min} /$ day vs. $19.2 \pm 18.6 \mathrm{~min} /$ day $)$ and marginally $(P=$ $0.080)$ more than normal controls $(25.8 \pm 23.4)$. There were no significant differences between the three groups in the amount of nonbout MVPA. These results provide further evidence that physical activity is important for long-term maintenance of weight loss and suggest that sustained volitional activity (i.e., $\geq 10 \mathrm{~min}$ in duration) may play an important role. Interventions targeting increases in structured exercise may be needed to improve long-term weight-loss maintenance.
\end{abstract}

\section{Introduction}

Given the critical role of physical activity in weight-loss maintenance, it is important to have objective data on the amount of physical activity common to individuals successful at weight-loss maintenance. The National Weight Control Registry (NWCR) was established 
in 1993 to investigate the characteristics and behaviors of individuals who have been successful at long-term weight-loss maintenance (1). With $\sim 6,000$ participants, the NWCR is the largest longitudinal prospective study of individuals successful at long-term maintenance of weight loss. To qualify for NWCR entry, individuals must have lost a minimum of $13.6 \mathrm{~kg}(30 \mathrm{lb})$ and have maintained that amount of weight loss for at least 1 year.

The physical activity habits of the initial 784 subjects who enrolled in the registry were described nearly 10 years ago in an analysis by Klem et al. (1). Initial registry entrants were predominantly female, white, married, and well educated. These initial entrants far exceeded the minimum criteria for registry entry: participants lost an average of $30 \mathrm{~kg}$ (66 lb) and maintained the minimum $13.6 \mathrm{~kg}$ weight loss for an average of 5.5 years. Physical activity seemed to be a key to success in this group — nearly $90 \%$ of entrants reported using a combination of diet plus exercise to both lose weight and maintain weight loss. On average, initial registry entrants reported expending $\sim 2,827 \mathrm{kcal} /$ week in physical activity_roughly the equivalent of walking 28 miles/week (1).

Activity data from this initial description of the NWCR have been referenced in several reviews and position statements regarding the role of physical activity in weight-loss maintenance (2-8). We recently re-examined the self-reported activity data for nearly 4,000 NWCR entrants and confirmed a high level of activity (mean \pm s.d. 2,621 $\pm 2,252 \mathrm{kcal} /$ week) in this larger sample (9). However, self-reported physical activity provides only crude estimates of levels of activity that are prone to overestimation. Recently, Phelan et al. (10) reported accelerometer-based estimates of physical activity in long-term successful weight losers vs. normal-weight controls and found that the former did significantly more physical activity per day (58.6 vs. $52.1 \mathrm{~min} /$ day). However, the study lacked an obese comparison group, so it remains unclear the extent to which this level of activity would be more extreme compared with obese controls. Moreover, the study did not examine the number and duration of exercise bouts. Given the well-recognized importance of activity in long-term weight control, objective data characterizing amount and type of activity in which successful weight-loss maintainers engage could be beneficial in clarifying activity recommendations for weight-loss maintenance.

Accelerometers designed for assessing human movement offer minute-by-minute measurements of activity and provide a means for quantifying overall movement over a period of several days to weeks. Unlike pedometers, which simply count steps, accelerometers can capture intensity, frequency, and duration of movement. Thus, they are able to distinguish moderate-to-vigorous physical activity (MVPA) from less-intense activity, and can distinguish sporadic MVPA from the more sustained MVPA that is usually defined as formal exercise.

The purpose of this study was to use accelerometers to objectively evaluate the amount and intensity of activity of individuals successful at long-term weight loss in the NWCR. We compared the amount and patterns of activity of NWCR subjects to a group of normalweight never-obese individuals and a group of overweight and obese individuals $\mathrm{We}$ 
hypothesized that NWCR subjects would engage in more sustained bouts of MVPA than normal-weight and overweight controls.

\section{Methods and Procedures}

\section{Participants}

Approval for the study was granted by the Colorado Multiple institutional review board and all subjects signed an informed consent form. NWCR subjects were recruited primarily from the NWCR database. We mailed a letter to all NWCR participants in the Denver area inviting them to participate. Control subjects were recruited through University of Colorado Denver e-mail and Internet publications. A brief description of the study's aim and procedures was provided in the advertisements (as required by our institutional review board) such that potential subjects were aware that study aims involved an assessment of physical activity with an activity monitor. Study advertisements asked interested persons to contact the research personnel over phone. Upon contact, subjects were screened for inclusion and exclusion criteria, and (if applicable) to ensure they still met NWCR criteria (maintenance of a $>13.6 \mathrm{~kg}$ weight loss for at least 1 year).

We compared NWCR subjects to two different control groups. The first was composed of a group of normal-weight individuals (BMI $\geq 18 \leq 25$ ) who had never been obese. These subjects were recruited to achieve a group mean BMI, which was similar to the mean current BMI of NWCR subjects. For simplicity, we termed this group normal-weight controls. The second group was composed of overweight and obese individuals (BMI $\geq 26 \leq 45$ ). These subjects were recruited to achieve a group mean BMI similar to the mean self-reported preweight-loss BMI of NWCR subjects. For simplicity, we termed this group overweight controls. Approximately, 120 potential subjects were screened to identify our control groups. Information about age, gender, and current BMI was collected and a nested subject selection procedure was used to match group means for gender and age and to achieve the desired group mean BMI matching. Though the NWCR is predominantly (>90\%) white, groups were not matched for ethnicity. Though we recruited primarily within the University of Colorado Denver, $40 \%$ of lean controls and $44 \%$ of overweight controls were not university employees. All subjects were Colorado residents.

We excluded individuals with physical or medical limitations that might prevent them from engaging in physical activity including cardiovascular disease, cerebrovascular disease, neurologic disease, active rheumatologic or musculoskeletal disease, chronic kidney or liver disease, untreated thyroid disorder, uncontrolled diabetes, active psychiatric illness, or substance abuse. We also screened potential subjects with a Physical Activity Readiness Questionnaire (11). We excluded pregnant and lactating women because their patterns of physical activity may be different from their usual baseline. We excluded individuals that reported swimming or water aerobics as their primary form of exercise as accelerometers cannot be worn in water and we would underestimate activity in these individuals. Subjects were not screened based on any other activity criteria. We excluded individuals who were not weight stable (loss or gain of $>10 \%$ of body weight in the past 6 months), who were actively trying to lose weight, or who had undergone weight-loss surgery. 


\section{Demographic and weight history}

Participants were asked to provide basic demographic information (age, gender, ethnicity, education level, and marital status) and details about weight history (maximum adult weight, duration of maintenance of required minimum $30 \mathrm{lb}$ weight loss).

\section{Height, weight, and BMI}

Height and weight were measured using a stadiometer and an electronic scale. BMI was calculated from the participants' height and weight (weight $(\mathrm{kg}) / \mathrm{height}^{2}(\mathrm{~m})$ )

\section{Measurement of physical activity with accelerometry}

Objective assessments of physical activity were obtained using the Stayhealthy RT3 (Stayhealthy, Monrovia, CA). Technical specifications of the device have been described elsewhere (12). Briefly, the RT3 is a triaxial accelerometer that uses a piezoelectric bender to detect acceleration of movement along three axes: vertical $(x)$, anteroposterior $(y)$, and mediolateral $(z)$ and covert them to activity counts. In addition, the device calculates a composite score for the overall vector magnitude calculated as vector magnitude $=\left(x^{2}+y^{2}+\right.$ $\left.z^{2}\right)^{0.5}$. Activity counts in each axis and the total vector magnitude activity counts are summed over a user-defined sampling period (epoch) and stored in the memory for subsequent retrieval. For this study, the epoch interval was set at 1 min (mode 3).

The RT3 has adequate intrainstrument and interinstrument coefficients of variation at the higher hertz frequencies (coefficients of variation $<10$ and $<2.5 \%$, respectively) when tested on a vibration table (12). The correlations between accelerometer counts and submaximal oxygen uptake for treadmill walking at different speeds and nonregulated physical activity are 0.79 and 0.89 , respectively (13).

The participant's height, age, and weight are required to initialize the RT3 unit. These measures were taken at a brief visit with the subject at the time the subject received the accelerometer. Standardized instructions for the activity monitor were provided to subjects. Subjects were asked to wear the accelerometer during all waking hours for a period of seven complete and consecutive days, with the exception of bathing and swimming. We asked that the week in which subjects wear the monitor should be "typical week", i.e. they should not be going on vacation or engaging in other unusual activities. Participants were asked to wear the unit on their waistband, and the location in which the unit was worn was to be consistent from day to day. The participant was not able to turn off the unit; and data were collected continuously by the device. In addition, the participant was not able to see activity counts recorded by the unit. The accelerometers were set to record continuously until their storage capacity in the mode selected (just over 7 days) was reached.

Testing was performed over a 2-year period between June 2004 and June 2006.

Accelerometer units were randomly assigned as recommended by Jakicic et al. (14) to minimize potential biases between the two groups due to any interinstrument differences that may exist. Equal numbers of NWCR subjects and controls were tested during any given 
calendar week in order to minimize the effect that time of year or weather may have on comparison of physical activity across groups.

\section{Data analysis}

File processing and data reduction-Data analysis was performed by computer programs in Visual Basic for Applications developed at the University of Colorado and the University of Pittsburgh. Total time the RT3 was worn was determined by subtracting nonwear time from total observation time for the day. Studies with other accelerometers have used variable definitions of nonwear time (15-17) and there is no clear consensus on how to define nonwear for accelerometers in general or for the RT3 in particular. As the RT3 has a wider frequency range than other accelerometers and our pilot data suggested sensitivity to ambient vibration, we chose to allow slightly more low-level activity counts in our definition of nonwear than the definitions that have been reported with other devices. We defined nonwear time for the RT3 as intervals of $>30$ consecutive minutes of zero counts with allowance for up to two consecutive minutes of observations of 1-100 counts/ min. Periods of nonwear were defined as ending when count levels exceeded zero counts for $>2$ consecutive minutes or when counts exceeded 100 counts/min.

Minimal wear parameters were chosen based on what has been reported necessary to achieve reasonable reliability for assessment of time spent in moderate-to-vigorous activity (18) and consistent with other studies using accelerometers to assess bouts of moderate-tovigorous physical activity (MVPA) $(15,19)$. A valid day was considered a day in which the device was worn for $>480 \mathrm{~min}(8 \mathrm{~h})$. Parameters were set to exclude from further analysis subjects who did not have at least 3 valid weekdays and 1 valid weekend day; however, no subjects failed to meet these criteria.

\section{Selection of MVPA cutpoints based on calibration studies of the RT3-The} intensity threshold for MVPA ( $\geq 984$ counts/min) was based on published activity count cutpoints for the RT3. Rowlands et al. (13) assessed the relationship between accelerometer counts and oxygen consumption during both treadmill walking/running and several nonregulated activities. They report a locomotor count cutpoint value for moderate activity (defined as $\geq 3$ metabolic equivalents) derived from assessment of treadmill activities ( $\geq 1,316.6$ counts $/ \mathrm{min}$ ), as well as a composite count cutpoint for moderate activity ( $\geq 984$ counts/min) based on all activities assessed. Because a primary objective in our study was to obtain an objective description of activity level and patterns in NWCR members, we chose the composite threshold of $\searrow 984$ counts/min as we felt it was more appropriate to the spectrum of activity in NWCR subjects. Although walking is a commonly reported activity in the NWCR (9), the majority (66\%) of NWCR members report participating in activities other than or in addition to walking (V.A. Catenacci, L.G. Ogden, J.O. Hill, and H.W. Wyatt, unpublished data). Other commonly reported activities in the NWCR include: resistance training (29.2\%), cycling (18.3\%), aerobics (15.7\%), cardiovascular exercise machines (14.6\%), and running (14\%) (ref. 9).

Measures of MVPA-We coded 1 min of RT3 data as 1 MVPA min if vector magnitude activity counts were $\$ 84$ counts/min. From these data, we calculated average daily minutes 
of MVPA in bouts and nonbouts. We defined a bout of activity as $\geq 10$ consecutive minutes of activity similar to parameters reported by prior studies including Strath et al. (15) and Troiano et al. (17) in the analysis of the NHANES 2003-2004 accelerometer data. We included a bout interruption allowance of 1 min of less than MVPA for every 9 min of MVPA activity as recommended when extracting bouts of MVPA (20). Nonbout activity was defined as episodes of MVPA lasting 1-9 min in duration. All accelerometer counts in the MVPA range were summed into either MVPA bout activity or MVPA nonbout activity. Our subsequent use of the term "bout MVPA" refers to MVPA that occurred as part of one of these concentrated bouts of activity, and "nonbout MVPA" to MVPA that occurred outside of these sustained bouts. We further examined patterns of activity by comparing the intensity, frequency, and duration of bout and nonbout MVPA between groups

\section{Statistical analysis}

Descriptive statistics are presented in tables as either mean \pm s.d. for continuous measures or percentages for categorical responses. Significance level was set at $\alpha=0.05$. Data were analyzed graphically to assess for any unusual values (none were found) and for normality. Although data presented in Table 2 (total time worn, total counts/day, and mean counts/min) were normally distributed, the data for MVPA bout and to a lesser extent nonbout minutes (Table 3) were not normally distributed. Thus, we have also reported the median and quartiles ( 25 and $75 \%$ points) for the bout and nonbout MVPA data. Independent sample $t$ tests were used to examine group differences in baseline demographic variables. $\chi^{2}$ Analyses were used to compare group proportions across categories. Independent sample $t$ tests were used to examine group differences in data that were normally distributed (total time worn, total accelerometer counts/day, and mean accelerometer counts/min).

Nonparametric Wilcoxon rank sum tests were used to examine group differences in data that were not normally distributed (bout and nonbout MVPA).

The sample size of this study was calculated to provide at least $80 \%$ power to detect an effect size of $10 \mathrm{~min} /$ day between groups in bout MVPA min/day based on a pooled standard deviation obtained from Jakicic et al. (21). However, the variability observed in this study was greater than was initially estimated. In a post hoc sample size calculation using a pooled standard deviation of $28.7 \mathrm{~min} /$ day we had $80 \%$ power to detect a difference of 21.4 bout MVPA min/day between groups.

\section{Results}

\section{Subject characteristics}

Subject characteristics are displayed in Table 1. We studied a total of 90 subjects: 26 NWCR, 30 normal-weight controls, and 34 overweight controls. Most subjects were female, reflecting the composition of the NWCR. The groups did not differ in age or marital status. Significantly more minority subjects were recruited in the normal-weight (20\%) and overweight (29\%) control groups as compared to the NWCR group (4\%). The current BMI of NWCR subjects $\left(23.4 \pm 2.5 \mathrm{~kg} / \mathrm{m}^{2}\right.$, mean \pm s.d. $)$, and of normal-weight controls ( $22.5 \pm$ $\left.1.6 \mathrm{~kg} / \mathrm{m}^{2}\right)$ was not significantly different $(P=0.149)$. The maximum BMI of NWCR subjects $\left(32.0 \pm 4.1 \mathrm{~kg} / \mathrm{m}^{2}\right)$ was also not significantly different from current BMI of 
overweight controls $\left(31.9 \pm 4.1 \mathrm{~kg} / \mathrm{m}^{2}\right), P=0.985$. NWCR subjects had lost a mean of 24.7 $\pm 9.6 \mathrm{~kg}$ (range 13.6-48.6 kg) and maintained the registry minimum $230 \mathrm{lb}(13.6 \mathrm{~kg}$ ) weight loss for $14.2 \pm 9.8$ years (range $2-37$ years). On average, subjects wore the physical activity monitor for $14.0 \pm 1.4 \mathrm{~h} /$ day and for $6.7 \pm 0.7$ days. There were no significant differences in monitor wear time between groups.

\section{Overall activity level}

Mean activity counts/min during the time the accelerometer worn are shown in Table 2 and were $334 \pm 114$ counts/min in NWCR, $312 \pm 142$ counts/min in normal-weight controls and $273 \pm 71$ counts/min in overweight controls. Differences in mean counts/min between groups were only significant $(P=0.012)$ for the comparison between NWCR and overweight controls. A similar pattern was observed with total accelerometer activity counts/day (Table 2).

\section{Patterns of MVPA}

Bout MVPA. NWCR subjects accumulated more minutes per day of MVPA in bouts of $\geq 10$ $\mathrm{min}$ than did overweight controls $(41.5 \pm 35.1 \mathrm{~min} /$ day vs. $19.2 \pm 18.6 \mathrm{~min} /$ day $)$. There was also a near significant trend $(P=0.080)$ for NWCR subjects to accumulate more daily minutes of bout MVPA than normal-weight controls $(25.8 \pm 23.4 \mathrm{~min} /$ day $)$. Similar results were seen for mean daily activity counts (Table 3 ). The mean number of bouts of MVPA $>10$ min was significantly higher $(P<0.05)$ in NWCR subjects $(1.4 \pm 1.0$ bouts/day) than in either normal-weight $(0.9 \pm 0.6$ bouts/day $)$ and overweight $(0.8 \pm 0.80$ bouts/day $)$ controls. NWCR subjects also had longer bouts and higher intensity bouts than overweight controls, but were similar to normal-weight controls on these variables (Table 3).

Nonbout MVPA. Mean daily minutes of MVPA occurring in bouts of $<10 \mathrm{~min}$ in duration (nonbout MVPA) were not significantly different across the three groups at $28.1 \pm 10.8$, $32.6 \pm 15.4$, and $32.3 \pm 12.4 \mathrm{~min} /$ day for NWCR, normal-weight and overweight controls, respectively. There were also no significant differences between the three groups in mean daily activity counts accumulated in nonbout MVPA, or in the frequency or duration of episodes of nonbout MVPA (Table 3). Mean intensity (counts/min) of nonbout MVPA is also shown in Table 3 and was significantly lower than bout MVPA in all three groups $(P<$ $0.001)$. Mean intensity of nonbout MVPA was slightly higher in normal-weight subjects $(1,379 \pm 80$ counts/min) and NWCR subjects $(1,371 \pm 80)$ as compared to overweight subjects $(1,344 \pm 88)$ but the difference was only significant for the comparison between normal-weight and overweight subjects $(P=0.037)$.

\section{Sensitivity analysis}

We repeated our analysis based on an MVPA threshold of $>1,316$ counts/min (threshold reported by Rowlands et al. (13) based on data for treadmill activities only) to determine the sensitivity to the count cutpoint we chose to use in our analysis (data not shown). We found that while the minutes of MVPA did decrease somewhat as would be expected (by 25-30\% for bout MVPA minutes and 40\% for nonbout MVPA minutes), the overall pattern of differences in minutes of bout and nonbout MVPA between the groups remained the same (including similar levels of statistical significance). 


\section{Discussion}

The results provide objective insight into the amount of activity that may be necessary to promote long-term weight-loss success. NWCR subjects engaged in $\sim 41.5 \mathrm{~min} / \mathrm{day}$ (or roughly $290 \mathrm{~min} /$ week) of sustained MVPA. This is significantly $>19.2 \mathrm{~min} /$ day (roughly $134 \mathrm{~min} /$ week) of sustained MVPA observed in obese controls. It was also greater (though not significantly so) than the $25.8 \mathrm{~min} /$ day (roughly $181 \mathrm{~min} /$ week) of sustained MVPA observed in normal-weight controls. These results, obtained with objective measures of physical activity, are consistent with previous measures of self-reported activity that suggest NWCR subjects are highly physically active $(9,22)$. The level of activity we found in successful weight-loss maintainers using objective measures is also similar to what has been shown to be necessary to prevent weight regain after weight loss in several prospective studies by Jakicic et al. $(21,23,24)$ in which level of activity was defined using self-report.

It is important to note that there is a great deal of variability in the amount of physical activity observed in all three groups, as reflected by relatively high standard deviation in bout and to a lesser extent nonbout MVPA minutes. It has previously been observed in the self-reported physical activity data in the NWCR (9) that there is considerable individual variability in the amount of activity reported. Our study objectively confirms substantial individual differences in the amount of MVPA observed in a group of successful weight-loss maintainers. Thus, it appears that some weight-reduced individuals can manage their weight with less activity and some may require more. The extent to which an individual is able to make and sustain changes in eating behaviors may influence the amount of activity needed to sustain weight loss. Other individual-specific factors such as amount of weight lost, age, gender, and genetic factors could also impact the amount of activity needed. For these reasons, it may be difficult to develop a single recommendation for the optimum amount of physical activity for weight-loss maintenance.

There is currently a wide range of physical activity that is recommended for maintaining weight loss. For example, the Dietary Guidelines for Americans 2005 (25) recommends 60$90 \mathrm{~min}$ of moderate-intensity physical activity per day (420-630 $\mathrm{min} /$ week) to sustain weight loss for previously overweight/obese people. Only $23.1 \%$ of NWCR subjects engaged in $>60 \mathrm{~min} / \mathrm{day}$ of bout MVPA and would therefore meet the recommended activity levels for this guideline. More recently the 2008 Physical Activity Guidelines for Americans (26) concluded that many adults will need to do $>150 \mathrm{~min} /$ week of moderate-intensity physical activity to lose and maintain weight loss, and some may need the equivalent of $2300 \mathrm{~min} /$ week of moderate-intensity physical activity to meet their weight-control goals. This guideline also recognizes that achieving energy balance and a healthy weight depends on both energy intake and energy expenditure and suggests that combined with restricting caloric intake; these adults should gradually increase minutes or intensity of activity until the physical activity is effective in achieving a healthy weight. Compared to these more recent recommendations, over two-thirds (69.2\%) of NWCR subjects in our study engage in $>150$ $\mathrm{min} /$ week of bout MVPA, and nearly one-third (30.8\%) were found to engage in $>300 \mathrm{~min} /$ week of bout MVPA. Thus, the recommendations outlined for individuals desiring to maintain weight loss in the 2008 Physical Activity Guidelines for Americans seem reasonable and consistent with the results of our study. The optimum amount of physical 
activity required for weight-loss maintenance will depend on energy intake and may also depend on other individual factors and will thus need to be adjusted on an individual basis.

The results of our study also suggest an important role for structured exercise in weight-loss maintenance. The patterns of physical activity observed in our study suggest that NWCR participants engage in purposeful, structured, exercise as reflected in minutes of MVPA accumulated in sustained bouts. Importantly, minutes of nonbout MVPA were similar across the three groups suggesting that outside of the exercise bouts, NWCR subjects were not any more or less active than their normal-weight and overweight counterparts at other times of the day. These results suggest that sustained activity (i.e. $\geq 10 \mathrm{~min}$ in duration) may be important for successful weight-loss maintenance. It may be that NWCR subjects engaged in planned exercise as a part of their weight-loss strategy, and continue to do so as they maintain their weight loss. Structured exercise may also be important because activity tends to be at a higher intensity during a sustained bout of activity. It is also possible that planned exercise is needed to achieve the amount of activity required by many individuals to sustain a weight loss.

We found some indication that NWCR subjects performed more exercise (as reflected by a trend for both higher bout MVPA minutes and higher activity counts accumulated in bouts of MVPA) than a group of normal-weight individuals; however, our sample size was not large enough to detect whether a difference of the magnitude observed was statistically significant. Our results in this area are consistent with a previous study by Phelan et al. (10), which compared activity patterns using the RT3 in a larger group of 135 individuals maintaining a $\geq 10 \%$ weight loss for at least 5 years as compared to 102 always normalweight controls. Though methods used to analyze the RT3 data were different (intensity cutoffs were based on metabolic equivalent levels calculated by the device, rather than activity counts), the results were similar. The long-term weight-loss maintainer group spent significantly more time per day than the always normal-weight group in MVPA. These results may suggest that in order to counter metabolic factors that predispose to weight regain (27-29), weight-reduced individuals require a greater level of physical activity to maintain their BMI than never-obese persons at the same BMI. Alternatively, the higher activity level of successful weight losers may be needed to offset greater caloric intake in these individuals. Although we did not assess dietary intake in this study, previous studies have suggested that successful weight losers report consuming significantly less fat and somewhat fewer calories than normal-weight individuals without a history of obesity (30).

A strength of this study is that it used objective measures rather than self-report to assess patterns of physical activity. The RT3 provides minute-by-minute data and allows detailed study of activity patterns not available from other more accurate but longer term cumulative measures such as doubly labeled water.

Our study did have some limitations. Accelerometers may underestimate the intensity of many common recreational activities other than walking and running on level surfaces (3134). Given the spectrum of activity expected in our NWCR subjects, we therefore chose to use the composite MVPA threshold ( $₫ 984$ counts/min) identified by Rowlands et al. (13), rather than the threshold defined based on locomotor activities only ( $\geq 1,316$ counts/day). 
However, it is possible that this composite threshold may have overestimated MVPA minutes in NWCR subjects or controls whose primary mode of activity was locomotor. We also used the same count cutpoint to identify MVPA in all three groups in our study. Chen $e t$ al. (35) have noted that to the extent that accelerometer counts may vary by BMI, MVPA estimates may be systematically biased in overweight and obese adults. The RT3 is a relatively new instrument and sufficient validation studies have not yet been conducted to identify appropriate count cutpoints that correspond to metabolic equivalent levels in a range of subjects. Additional validation studies are needed to determine population specific MVPA cutpoints for the RT3. Future studies with more sophisticated methods of measuring energy expenditure (i.e. doubly labeled water) are also recommended to further characterize activity levels in these populations.

Our study results may not be generalizable to men or to certain ethnic groups. Our subjects were predominantly (nearly $90 \%$ ) female. Although current physical activity recommendations do not differ between men and women, prior research in the NWCR $(1,9)$ suggests that there may be differences in the magnitude of physical activity reported by weight-loss maintainers based on gender. Studies are needed to examine physical activity patterns in men maintaining a significant weight loss to determine whether the results of this study can be generalized to this group. In addition, groups were not matched for ethnicity. Our control groups had significantly more ethnic minority subjects than our NWCR group which could have biased results given data showing ethnic differences in activity levels $(17,19)$.

The results from this study may also not be representative of the activity levels of weightreduced, normal-weight, or overweight adults in the US population as a whole. The normalweight and overweight control groups in our study appeared more active than previously reported national averages for engagement in bout MVPA. Data from the NHANES 20032004 physical activity monitoring component (15) comparing bouts ( $\geq 10 \mathrm{~min}$ ) of MVPA by BMI category found mean bout MVPA minutes per day were 18.0 in the normal-weight group, 16.0 in the overweight subjects, and 10.3 in obese subjects, using the Actigraph AM-7164 accelerometer (ActiGraph LLC, Fort Walton Beach, Florida). Differences in the duration of interruption allowed when extracting bouts of activity may in part explain these findings. Our analysis allowed 1 min of less than MVPA for every 9 min of MVPA activity, while no bout interruptions allowances were included in the analysis of the NHANES data published by Strath et al. (15) Our screening procedures also excluded a broad range of medical conditions, which might interfere with level of activity and it is possible that the general good health of our subjects may in part explain why higher levels of activity were observed in our controls. Additional factors related to recruitment strategies (volunteers were aware that the study involved an assessment of physical activity) and study location (Colorado is one of the leanest states in the nation (36) and may have a more physically active population (37)) may also have resulted in higher levels of activity in all three groups.

In summary, on average, individuals successful at weight-loss maintenance spent significantly more total time each day in structured activity (bout MVPA) than their overweight and obese controls and tended to engage in more structured activity as compared with their never-obese normal-weight controls. Findings from this study provide further 
evidence that high levels of physical activity are important for long-term maintenance of weight-loss and support the concept that a greater duration of physical activity may be needed for weight regain prevention than for weight-gain prevention. The activity patterns observed in our study also suggest that structured activity may play an important role in weight-loss maintenance, and interventions targeting increases in structured exercise may be needed to improve long-term weight-loss maintenance. It is also important to note there was a great deal of variability in the amount of physical activity observed in successful weightloss maintainers. Over two-thirds of NWCR subjects in our study engage in $>150 \mathrm{~min} /$ week of bout MVPA, and about one-third were found to engage in $>300 \mathrm{~min} /$ week of bout MVPA. This may reflect that there is a range of physical activity that may be required to regulate body weight that will depend on energy intake and may also be impacted by other individual factors. As suggested in the 2008 Physical Activity Guidelines for Americans (26), physical activity goals for weight-loss maintenance should be adjusted on an individual basis. Future studies designed to obtain a better understanding of the individual-specific determinants of how much activity is required for weight-loss maintenance, including the role of dietary factors, should be a high research priority.

\section{REFERENCES}

1. Klem ML, Wing RR, McGuire MT, Seagle HM, Hill JO. A descriptive study of individuals successful at long-term maintenance of substantial weight loss. Am J Clin Nutr. 1997; 66:239-246. [PubMed: 9250100]

2. Hill JO, Wyatt HR. Role of physical activity in preventing and treating obesity. J Appl Physiol. 2005; 99:765-770. [PubMed: 16020440]

3. Donnelly JE, Smith B, Jacobsen DJ, et al. The role of exercise for weight loss and maintenance. Best Pract Res Clin Gastroenterol. 2004; 18:1009-1029. [PubMed: 15561636]

4. Jakicic JM. Exercise in the treatment of obesity. Endocrinol Metab Clin North Am. 2003; 32:967980. [PubMed: 14711070]

5. Jakicic JM, Clark K, Coleman E, et al. American College of Sports Medicine. American College of Sports Medicine position stand. Appropriate intervention strategies for weight loss and prevention of weight regain for adults. Med Sci Sports Exerc. 2001; 33:2145-2156. [PubMed: 11740312]

6. Jakicic JM, Otto AD. Physical activity considerations for the treatment and prevention of obesity. Am J Clin Nutr. 2005; 82:226S-229S. [PubMed: 16002826]

7. Saris WH, Blair SN, van Baak MA, et al. How much physical activity is enough to prevent unhealthy weight gain? Outcome of the IASO $1^{\text {st }}$ Stock Conference and consensus statement. Obes Rev. 2003; 4:101-114. [PubMed: 12760445]

8. Votruba SB, Horvitz MA, Schoeller DA. The role of exercise in the treatment of obesity. Nutrition. 2000; 16:179-188. [PubMed: 10705072]

9. Catenacci VA, Ogden LG, Stuht J, et al. Physical activity patterns in the National Weight Control Registry. Obesity (Silver Spring). 2008; 16:153-161. [PubMed: 18223628]

10. Phelan S, Roberts M, Lang W, Wing RR. Empirical evaluation of physical activity recommendations for weight control in women. Med Sci Sports Exerc. 2007; 39:1832-1836. [PubMed: 17909412]

11. Thomas S, Reading J, Shephard RJ. Revision of the Physical Activity Readiness Questionnaire (PAR-Q). Can J Sport Sci. 1992; 17:338-345. [PubMed: 1330274]

12. Powell SM, Jones DI, Rowlands AV. Technical variability of the RT3 accelerometer. Med Sci Sports Exerc. 2003; 35:1773-1778. [PubMed: 14523319]

13. Rowlands AV, Thomas PW, Eston RG, Topping R. Validation of the RT3 triaxial accelerometer for the assessment of physical activity. Med Sci Sports Exerc. 2004; 36:518-524. [PubMed: 15076796] 
14. Jakicic JM, Winters C, Lagally K, et al. The accuracy of the TriTrac-R3D accelerometer to estimate energy expenditure. Med Sci Sports Exerc. 1999; 31:747-754. [PubMed: 10331898]

15. Strath SJ, Holleman RG, Ronis DL, Swartz AM, Richardson CR. Objective physical activity accumulation in bouts and nonbouts and relation to markers of obesity in US adults. Prev Chronic Dis. 2008; 5:A131. [PubMed: 18793519]

16. Matthews CE, Chen KY, Freedson PS, et al. Amount of time spent in sedentary behaviors in the United States, 2003-2004. Am J Epidemiol. 2008; 167:875-881. [PubMed: 18303006]

17. Troiano RP, Berrigan D, Dodd KW, et al. Physical activity in the United States measured by accelerometer. Med Sci Sports Exerc. 2008; 40:181-188. [PubMed: 18091006]

18. Matthews CE, Ainsworth BE, Thompson RW, Bassett DR Jr. Sources of variance in daily physical activity levels as measured by an accelerometer. Med Sci Sports Exerc. 2002; 34:1376-1381. [PubMed: 12165695]

19. Young DR, Jerome GJ, Chen C, Laferriere D, Vollmer WM. Patterns of physical activity among overweight and obese adults. Prev Chronic Dis. 2009; 6:A90. [PubMed: 19527591]

20. Mâsse LC, Fuemmeler BF, Anderson CB, et al. Accelerometer data reduction: a comparison of four reduction algorithms on select outcome variables. Med Sci Sports Exerc. 2005; 37:S544S554. [PubMed: 16294117]

21. Jakicic JM, Polley BA, Wing RR. Accuracy of self-reported exercise and the relationship with weight loss in overweight women. Med Sci Sports Exerc. 1998; 30:634-638. [PubMed: 9565948]

22. Klem ML, Wing RR, Chang CC, et al. A case-control study of successful maintenance of a substantial weight loss: individuals who lost weight through surgery versus those who lost weight through non-surgical means. Int J Obes Relat Metab Disord. 2000; 24:573-579. [PubMed: 10849578]

23. Jakicic JM, Marcus BH, Gallagher KI, Napolitano M, Lang W. Effect of exercise duration and intensity on weight loss in overweight, sedentary women: a randomized trial. JAMA. 2003; 290:1323-1330. [PubMed: 12966123]

24. Jakicic JM, Marcus BH, Lang W, Janney C. Effect of exercise on 24-month weight loss maintenance in overweight women. Arch Intern Med. 2008; 168:1550-1559. discussion 1559. [PubMed: 18663167]

25. [Accessed 28 July 2010] U.S. Department of Agriculture Center for Nutrition Policy and Promotion Dietary Guidelines for Americans 2005. <http://www.cnpp.usda.gov/Publications/ DietaryGuidelines/2005/2005DGPolicyDocument.pdf $>$.

26. [Accessed 28 July 2010] U.S. Department of Health and Human Services 2008 Physical Activity Guidelines for Americans. 〈http://www.health.gov/paguidelines/pdf/paguide.pdf>.

27. MacLean PS, Higgins JA, Jackman MR, et al. Peripheral metabolic responses to prolonged weight reduction that promote rapid, efficient regain in obesity-prone rats. Am J Physiol Regul Integr Comp Physiol. 2006; 290:R1577-R1588. [PubMed: 16455763]

28. MacLean PS, Higgins JA, Johnson GC, et al. Enhanced metabolic efficiency contributes to weight regain after weight loss in obesity-prone rats. Am J Physiol Regul Integr Comp Physiol. 2004; 287:R1306-R1315. [PubMed: 15331386]

29. MacLean PS, Higgins JA, Johnson GC, et al. Metabolic adjustments with the development, treatment, and recurrence of obesity in obesity-prone rats. Am J Physiol Regul Integr Comp Physiol. 2004; 287:R288-R297. [PubMed: 15044180]

30. Phelan S, Lang W, Jordan D, Wing RR. Use of artificial sweeteners and fat-modified foods in weight loss maintainers and always-normal weight individuals. Int J Obes (Lond). 2009; 33:11831190. [PubMed: 19636318]

31. Hendelman D, Miller K, Baggett C, Debold E, Freedson P. Validity of accelerometry for the assessment of moderate intensity physical activity in the field. Med Sci Sports Exerc. 2000; 32:S442-S449. [PubMed: 10993413]

32. Bouten CV, Westerterp KR, Verduin M, Janssen JD. Assessment of energy expenditure for physical activity using a triaxial accelerometer. Med Sci Sports Exerc. 1994; 26:1516-1523. [PubMed: 7869887] 
33. Nichols JF, Morgan CG, Sarkin JA, Sallis JF, Calfas KJ. Validity, reliability, and calibration of the Tritrac accelerometer as a measure of physical activity. Med Sci Sports Exerc. 1999; 31:908-912. [PubMed: 10378921]

34. Bassett DR Jr. Validity and reliability issues in objective monitoring of physical activity. Res Q Exerc Sport. 2000; 71:S30-S36. [PubMed: 10925822]

35. Chen C, Jerome GJ, Laferriere D, Young DR, Vollmer WM. Procedures used to standardize data collected by RT3 triaxial accelerometers in a large-scale weight-loss trial. J Phys Act Health. 2009; 6:354-359. [PubMed: 19564665]

36. Centers for Disease Control and Prevention. U.S. Obesity Trends (Trends by State 1985-2008 March 24, 2010. <http://www.cdc.gov/obesity/data/trends.html\#state>.

37. Wyatt HR, Peters JC, Reed GW, Barry M, Hill JO. A Colorado statewide survey of walking and its relation to excessive weight. Med Sci Sports Exerc. 2005; 37:724-730. [PubMed: 15870624] 


\section{로을}

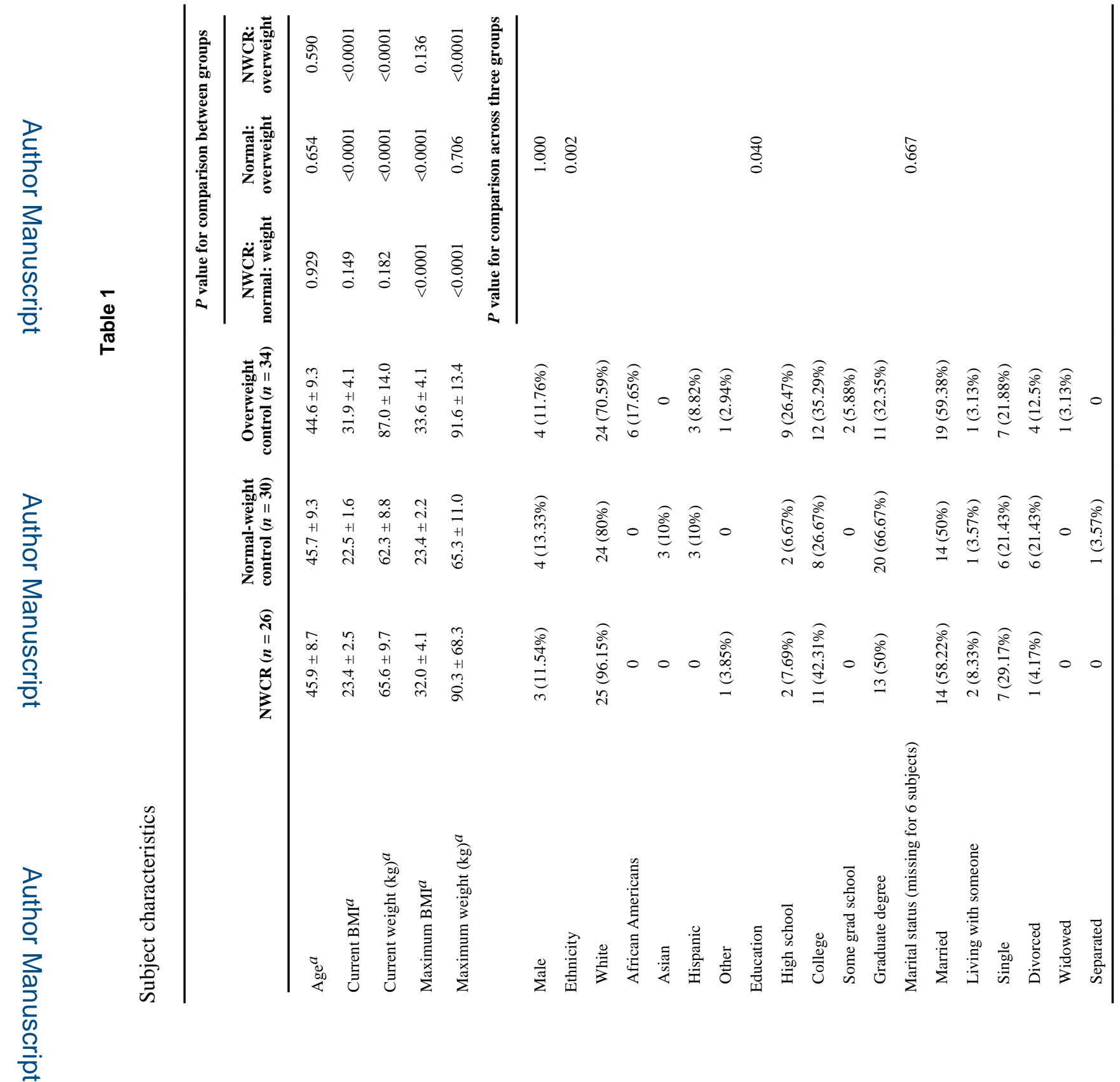

Obesity (Silver Spring). Author manuscript; available in PMC 2015 September 04. 

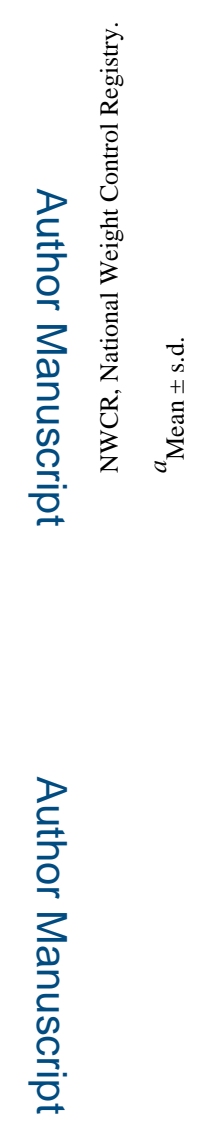

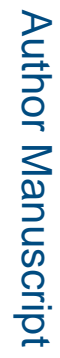

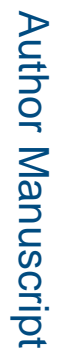

Obesity (Silver Spring). Author manuscript; available in PMC 2015 September 04. 


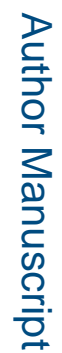

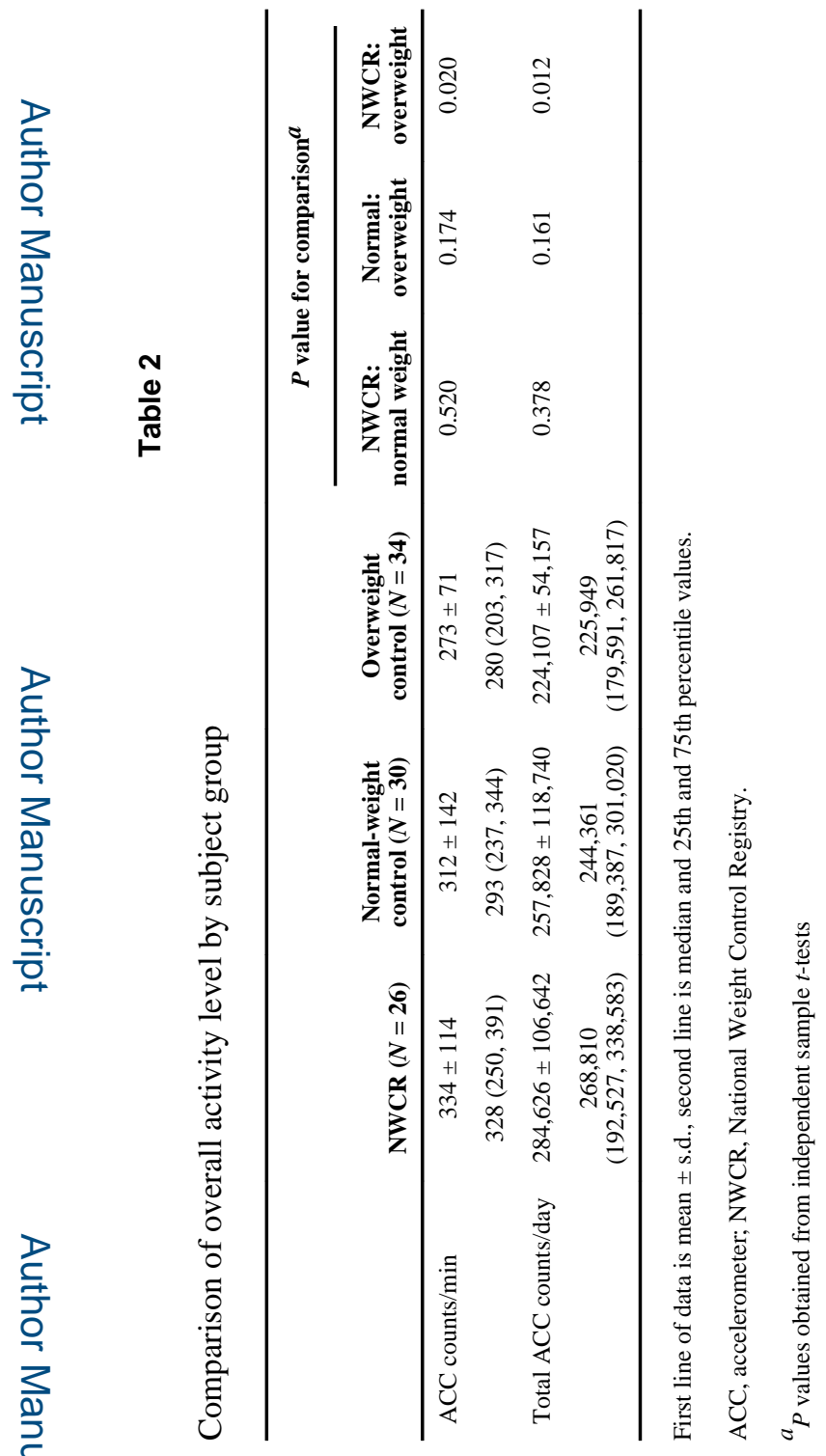




\section{로을}

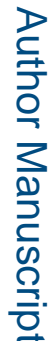

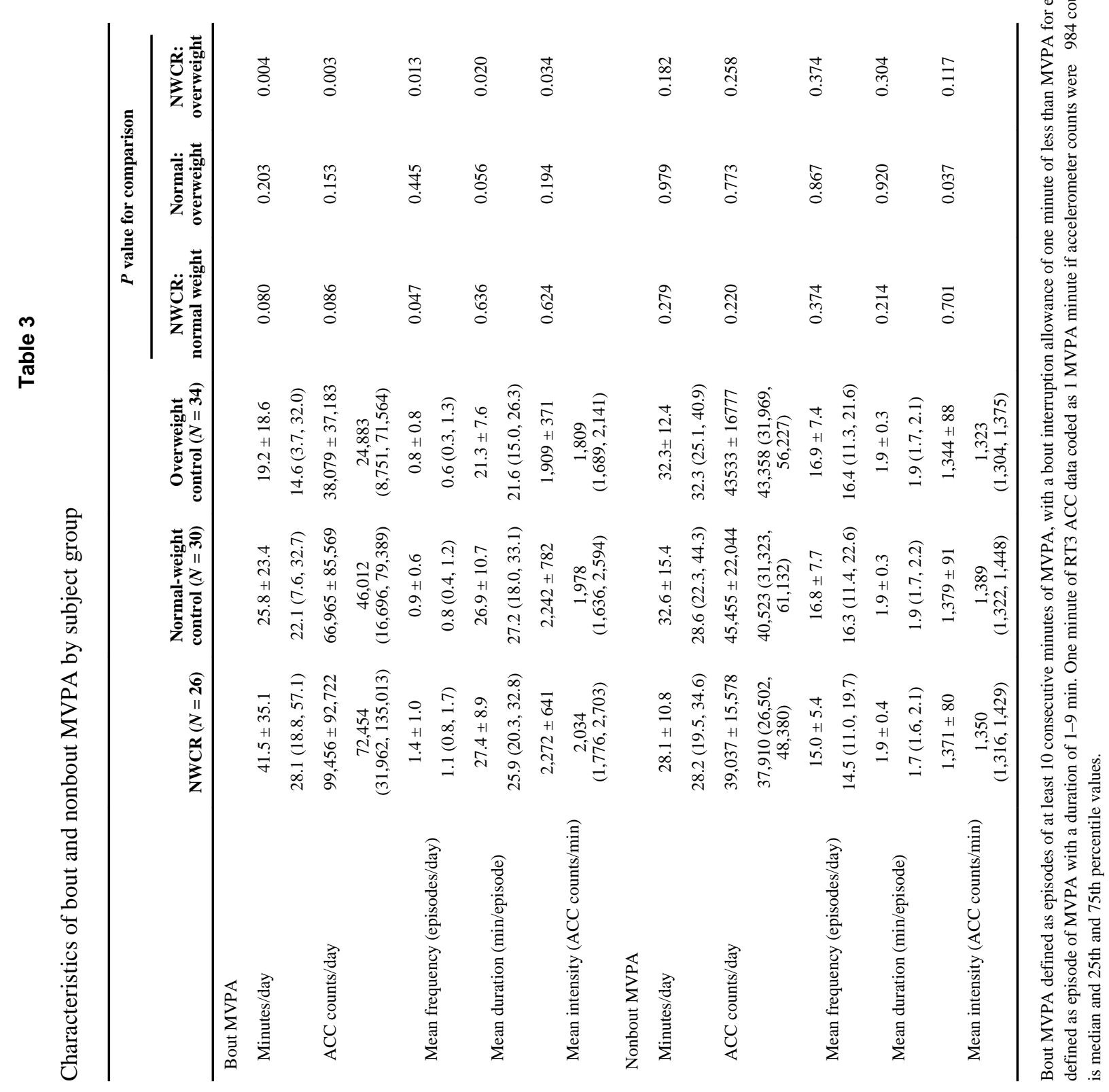

Obesity (Silver Spring). Author manuscript; available in PMC 2015 September 04. 


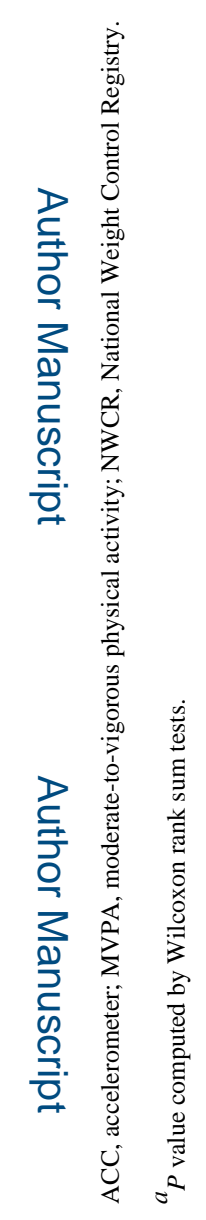

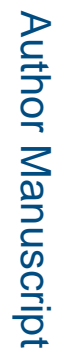

를

Obesity (Silver Spring). Author manuscript; available in PMC 2015 September 04. 